\title{
Polarization Rotation and Mode Splitting in Photonic Crystal Line-Defect Waveguides
}

\author{
Moïse Sotto ${ }^{1}$, Isao Tomita ${ }^{1,2}$, Kapil Debnath ${ }^{1,3}$ and Shinichi Saito ${ }^{1 *}$ \\ 1 Sustainable Electronic Technologies, Department of Electronics \& Computer Science, Faculty of Physical Sciences and \\ Engineering, University of Southampton, Southampton, United Kingdom, ${ }^{2}$ Department of Electrical \& Computer Engineering, \\ National Institute of Technology, Gifu College, Motosu, Japan, ${ }^{3}$ Electronics \& Electrical Communication Engineering, Indian \\ Institute of Technology, Kharagpur, India
}

In a line-defect waveguide of a planer photonic crystal (PhC), we found a new rotational state of polarized light, which exhibits "polarization rotation" on the PhC plane, when a phase mismatch $m$ was added to the air-hole alignment of the waveguide, where mode splitting was simultaneously observed in the dispersion curve. To account for the polarization rotation together with the mode splitting, we propose a two-state model that is constructed from Schrödinger equation obtained from the equation for electromagnetic waves. The proposed two-state model gives an explanation on the

OPEN ACCESS

Edited by:

Michael Mazilu,

University of St Andrews,

United Kingdom

Reviewed by:

Sebastian A. Schulz,

University of St Andrews,

United Kingdom

Venu Gopal Achanta,

Tata Institute of Fundamental

Research, India

*Correspondence:

Shinichi Saito

S.Saito@soton.ac.uk

Specialty section:

This article was submitted to

Optics and Photonics,

a section of the journal

Frontiers in Physics

Received: 01 May 2018

Accepted: 23 July 2018

Published: 14 August 2018

Citation:

Sotto M, Tomita I, Debnath K and Saito S (2018) Polarization Rotation and Mode Splitting in Photonic Crystal

Line-Defect Waveguides.

Front. Phys. 6:85

doi: 10.3389/fphy.2018.00085 relation between the polarization-rotational angle $\theta$ and the mismatch $m$ and on its rotational direction (i.e., clockwise or anticlockwise direction) that depends on the mode. Using the two-state model, we also discuss the angular momenta of the polarized light in the PhC waveguide, which are directly related to the Stokes parameters that characterize the polarization rotations.

Keywords: silicon photonics, polarization, photonic crystal, phase mismatch, Stokes parameters

\section{INTRODUCTION}

In a line-defect waveguide of a planer photonic crystal $(\mathrm{PhC})$, the polarization of light can rotate on the two dimensional $\mathrm{PhC}$ plane by addition of a phase mismatch to the air-hole alignment of the waveguide, which occurs without non-linear optical interactions (e.g., optical Kerr effects and photorefractive effects). Originally, the presence of light rotation, given as an optical vortex that carries angular momentum, in photonic bandgap media [1-4] via such non-linear interactions has been found in theoretical and experimental investigations [5-10]; This mechanism is attributed to the localized vortex state induced by those non-linear effects in the bandgap media, which is thus sometimes called a gap vortex. This is an analogous concept to a gap soliton in the bandgap media $[11,12]$.

However, without such non-linear effects, we can show the presence of a rotational state of polarized light just by adding the mismatch $m(0<m<a)$ to the air-hole alignment of a sidewall of the waveguide [13], as illustrated in Figure 1, where $a$ is the air-hole period of the PhC waveguide. Mock et al. also studied the same waveguide [14], but did not report such rotation. They just reported that the light path in the waveguide was "zigzag." An interesting feature of the rotation that we found is that if light propagates from a non-mismatched region to the mismatched region with a gradual mismatch change between them, the light polarization gradually rotates. Here we can define its rotational angle $\theta$ for a given $m$, and as will be shown later, $\theta$ is proportional to $m$ (for small $m$ compared with $a$ ) via numerical simulations. 


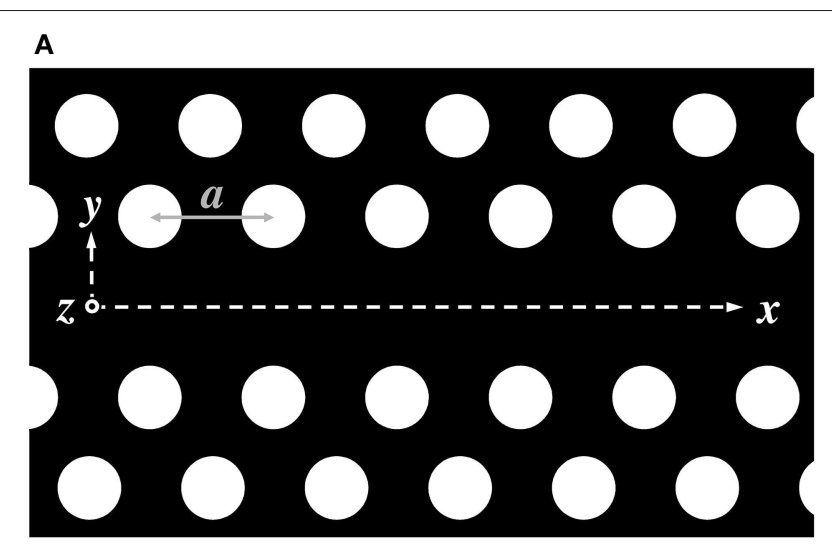

B

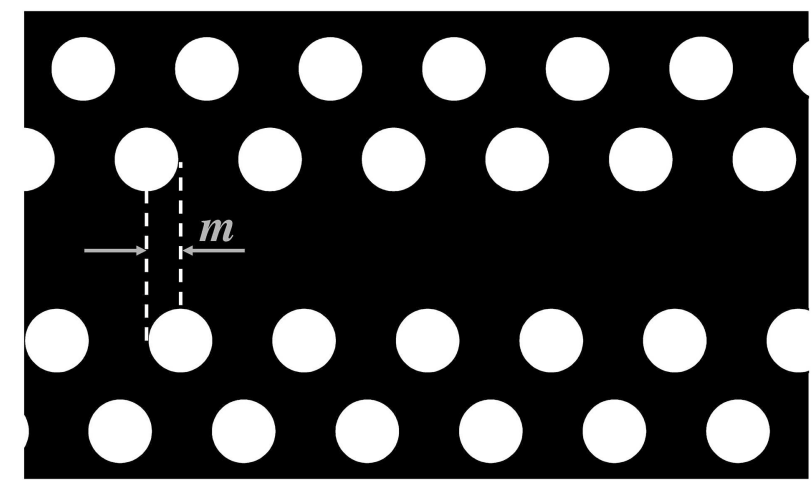

FIGURE 1 | PhC line-defect waveguides, where light propagates through the central part in the $x$-direction. The white circles are air-holes made on a black material (e.g., silicon), where the air-hole spacing (or lattice constant) is a, and the air-hole diameter is assumed to be smaller than a. (A) Air-hole alignments are symmetric with respect to the central line (or the $x$-axis). (B) The lower part of the air-hole alignment is shifted with the addition of a mismatch $m$, which causes the polarization rotation of light.

Moreover, with the addition of $m$, mode splitting (or level splitting) is observed in the dispersion curve [13, 14], which is created from an originally degenerated mode that contains different polarizations. The main cause of this mode splitting is symmetry breaking; When $m=0$, structural symmetry in the $x$ and $y$ directions is maintained (despite the presence of a linedefect waveguide at $y=z=0$ ), which causes highly degenerated states in the dispersion curve. But, structural symmetry breaking caused by $m \neq 0$ can resolve the degenerated states, creating two split modes, as will be shown in detail in section 2 .

We infer from our numerical simulations that the polarization rotation is intimately connected with the mode splitting, because they occur simultaneously, triggered by the addition of $m$. (Because of the above reasons, the mechanism of the rotational light obtained in our research is completely different from that of a gap vortex with non-linear interactions; the rotation that we found is spatial rotation, not temporal rotation seen for the localized vortex.)

In this paper, we will gain a qualitative understanding of the simultaneous polarization rotation and mode splitting from our

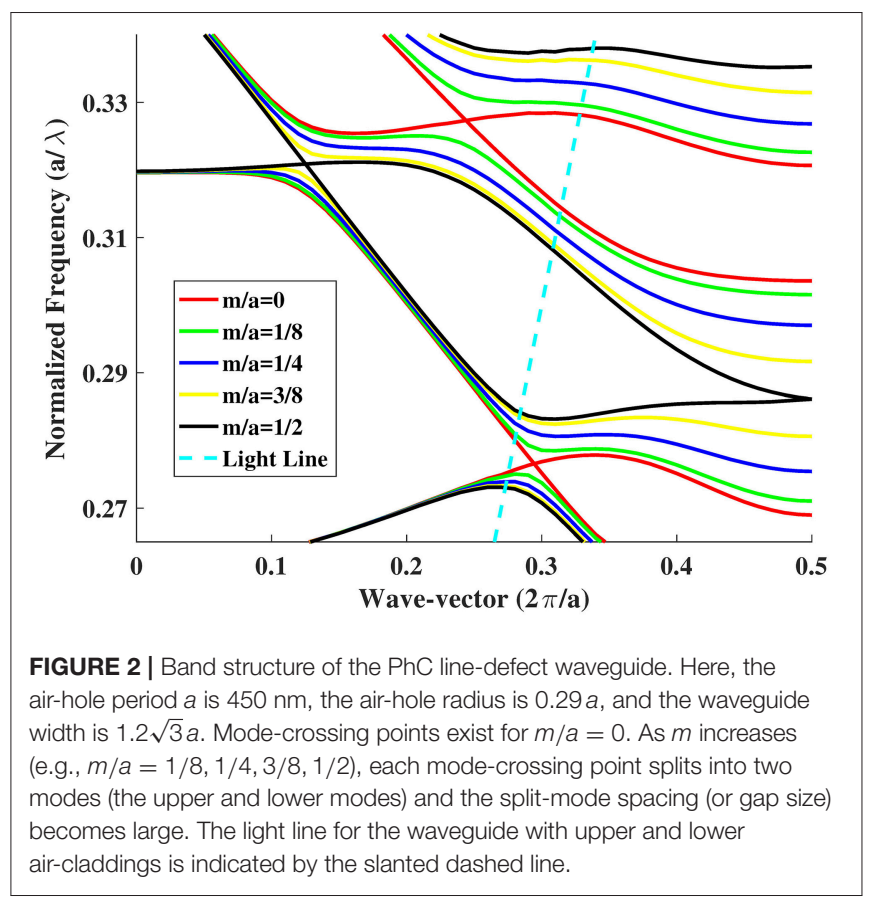

two-state model constructed via Schrödinger equation derived from the equation for electromagnetic (EM) waves in the waveguide. In this analysis, we can show that there is a relation between the polarization-rotational angle $\theta$ and the mismatch $m$ (via a relation between the mode-splitting spacing $\omega$ and the mismatch $m$ ), which is an analogous relation to that between an electron-energy-level splitting $\hbar \omega$ and an applied magnetic field $B$, or $\hbar \omega \propto B$.

In the next section, we will give some numerical simulation results, and then introduce a theory that connects the mode splitting with the polarization rotation within linear optics.

\section{NUMERICAL RESULTS FOR MODE SPLITTING AND POLARIZATION ROTATION}

Finite-difference time-domain computations $[13,14]$ show that when $m=0$, the band structure (or the dispersion curve) of a $\mathrm{PhC}$ line-defect waveguide has mode-crossing points, as depicted in Figure 2. Each of the mode-crossing points consists of two degenerated modes with different polarizations, which are yielded by band-folding (realized in the reduced zone for the wavenumber $k$ at $-\pi / a \leq k \leq \pi / a$ ). With the addition of non-zero $m$, the two-fold degeneracy is resolved (see Figure 2), thereby creating two split modes, where the gap size $f$ between the split modes increases with increasing $m$. (Schematic diagrams of Figure 3 well describe this behavior, and show that the mode splitting can be regarded as level splitting). The electric fields with different polarizations (with even or odd modes) come to what we call $\mathrm{TE}_{1}^{-}$or $\mathrm{TE}_{1}^{+}$, respectively, as displayed in Figure 4 for $k=0.2925 \times 2 \pi / a$ at $m / a=0,1 / 8,1 / 4,3 / 8,1 / 2$. (Much 

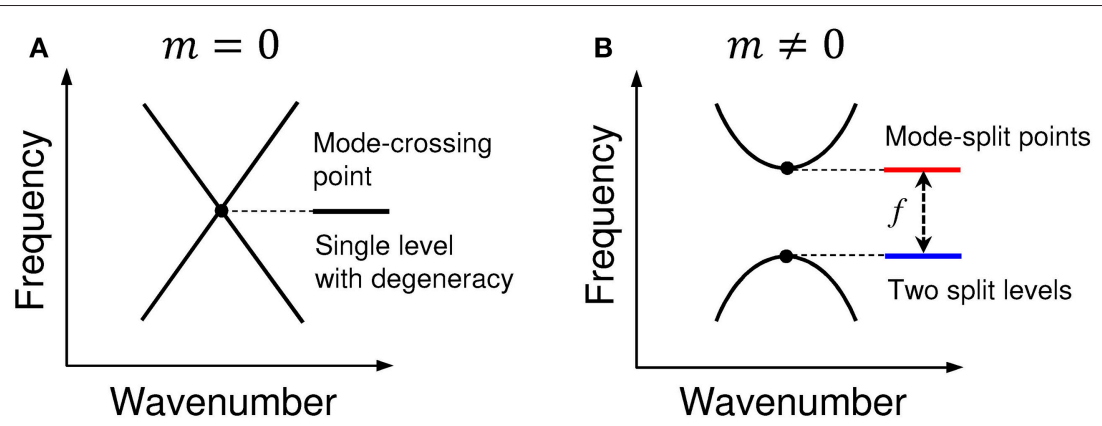

FIGURE 3 | Schematic diagrams for (A) a mode-crossing point with $m=0$, which can be regarded as a single level with degeneracy, and (B) mode-split points with $m \neq 0$, which can be looked upon as two split levels.

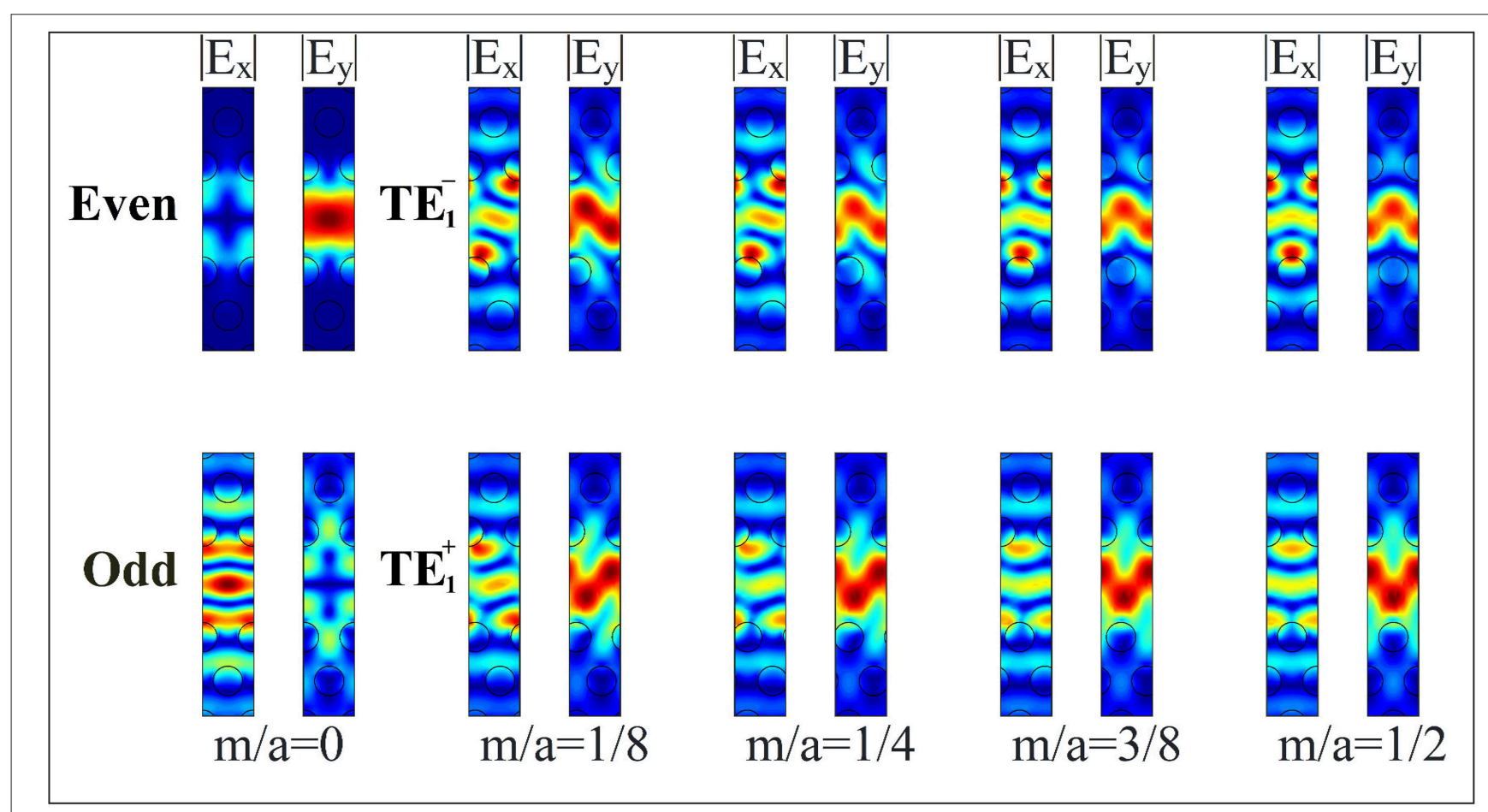

FIGURE 4 | Electric fields with different polarizations that come to the $\mathrm{TE}_{1}^{-}$- and $\mathrm{TE}_{1}^{+}$-polarizations with even and odd modes, respectively, for $m / a=0,1 / 8,1 / 4,3 / 8$, $1 / 2$ at $k=0.2925 \times 2 \pi / a$. Here, the absolute value of $E_{x}$ and $E_{y}$ is plotted.

clearer polarization rotation can be seen in $H_{z}$, and this will also be shown below.)

Figure 5 with the blue $\left(\mathrm{TE}_{1}^{+}\right)$and red $\left(\mathrm{TE}_{1}^{-}\right)$curves depicts a calculated $m$-dependence of the split modes, $\mathrm{TE}_{1}^{+}$and $\mathrm{TE}_{1}^{-}$, that correspond to those in Figure 3B, which are produced from the lowest degenerated $\mathrm{TE}_{1}$ mode at $190.13 \mathrm{THz}$ with $k=0.2925 \times$ $2 \pi / a$. Here, we chiefly focus on the lowest mode $\left(\mathrm{TE}_{1}\right)$ because its crossing point at $190.13 \mathrm{THz}$ with $k=0.2925 \times 2 \pi / a$ is placed inside the light cone, that is, the light at that point is a reallypropagating mode, not a radiation mode. Also, we observed that a maximum gap size $f_{\max }=8.68 \mathrm{THz}$ was obtained at $m / a=0.5$ and that the splitting behavior was symmetric for (a) $0<m / a \leq$ 0.5 and (b) $0.5<m / a<1$. Thus we plotted the figure for Case (a) only, which is sufficient to look at the essence of the behavior. The inset of Figure 5 shows the $m$-dependence of the normalized gap size, $f / f_{\max }$. Here, we phenomenologically found that it had good linearity, i.e., $f / f_{\max }=g m / a(g=2.5615)$ at $m / a \lesssim 0.3$ (but $f / f_{\max }$ leveled off near $m / a=0.5$ ). The $g=2.5615$ was obtained via the least square fitting with a straight line for the data points at $m / a$ less than or equal to 0.2875 . Note that we also observed that the center (say $\left.\bar{\Omega}=\left(\Omega_{0}+\Omega_{1}\right) / 2\right)$ of the $\mathrm{TE}_{1}^{+}$and $\mathrm{TE}_{1}^{-}$modes (say $\Omega_{0}$ and $\Omega_{1}$ ) showed little $m$ dependence (almost no $m$-dependence), as indicated by the black dashed curve in Figure 5 (although $\Omega_{1}-\Omega_{0}=\omega=2 \pi f$ showed a large $m$-dependence, as seen in Figure 5).

We then give clearer field and intensity profiles of the $\mathrm{TE}_{1}^{-}$ and $\mathrm{TE}_{1}^{+}$modes for $H_{z}$ : Figure $6 \mathbf{A}$ shows the field profile or the real part of $H_{z}$ in a unit cell at $k=\pi / a$ of the Brillouin-zone 


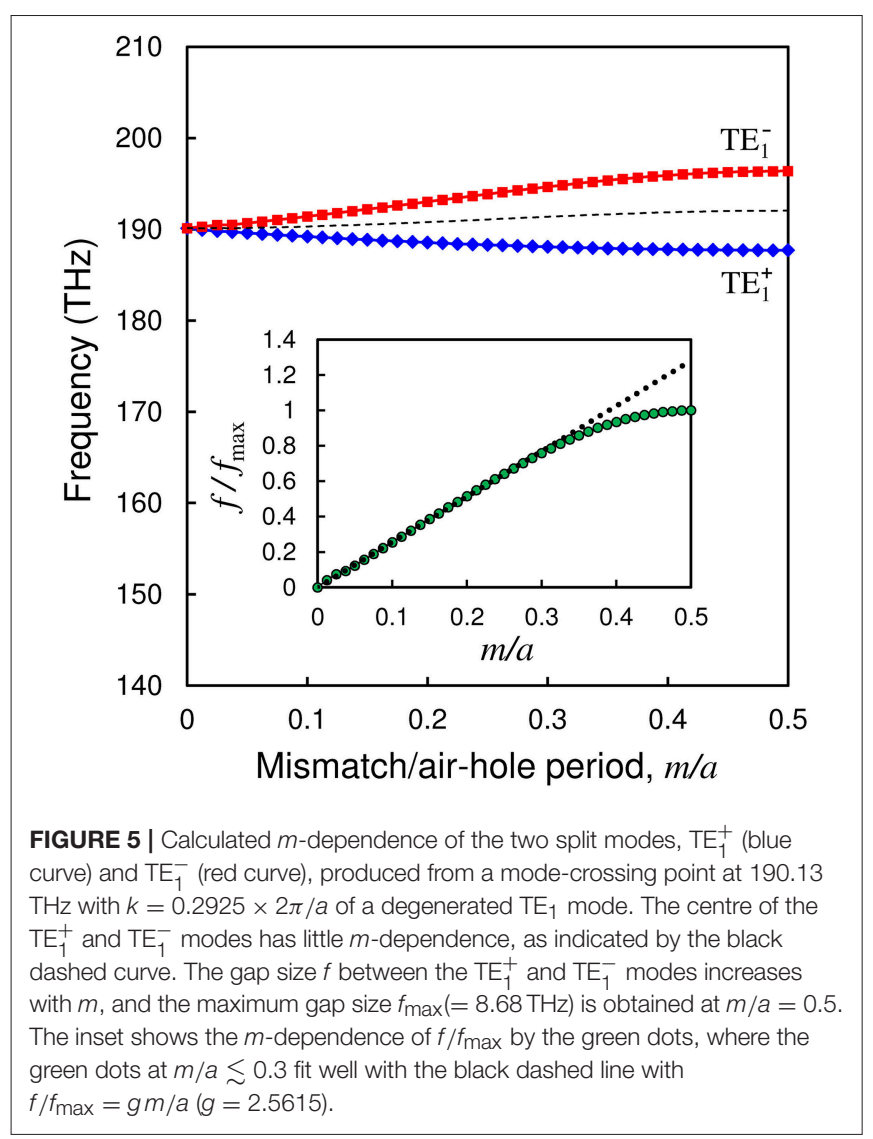

(BZ) end, and Figure $\mathbf{6 B}$ shows the intensity profile $\left|H_{z}\right|^{2}$ at a mode-crossing point, $k=0.2925 \times 2 \pi / a$. We then observe clearer tilted field and intensity profiles with a tilted angle (or a rotational angle) $\theta$ that increases with increasing $m$. In this case, we found that the rotation of the intensity profiles of $\mathrm{TE}_{1}^{+}\left(\mathrm{TE}_{1}^{-}\right)$ in Figure 6B was in the anticlockwise (clockwise) direction; A similar tendency remained in the field rotations of Figure 6A at the $\mathrm{BZ}$ end (since we observed in numerical simulations that the mode-splitting spacing became large even at the $\mathrm{BZ}$ end as $m$ increased).

The above results were obtained only from the numerical simulations (i.e., with no theoretical explanations via analytical methods). In the next section, we will explain the observed simultaneous polarization rotation and mode splitting by use of an analytical method with a two-state model derived from the equation for EM waves.

\section{ANALYTICAL INTERPRETATION AND DISCUSSION}

To derive the two-state model to account for the above phenomena, we start with the following wave equation (obtained from Maxwell's equations) for light propagating in the $x$ direction with polarization $E_{i}$ :

$$
\left(\nabla^{2}-\frac{n^{2}}{c^{2}} \frac{\partial^{2}}{\partial t^{2}}\right) E_{i}=0
$$

where $\nabla^{2}=\partial^{2} / \partial x^{2}+\partial^{2} / \partial y^{2}+\partial^{2} / \partial z^{2}, n$ is the refractive index that includes the $\mathrm{PhC}$ spatial modulation, and $c$ is the velocity of light.

In the following, we make an approximation that the inputpulse spatial-width $W(>\lambda)$ is larger than the air-hole period $a$ in the waveguide, where the input wavelength $\lambda$ also needs to suffices $\lambda>a$. This approximation makes it possible to deal with the phenomenon (light rotation) analytically, but cannot well describe it near the $\mathrm{PhC}$ band edge because the backward reflection of propagating light is strong due to Bragg reflection with the air-hole arrays. Within the above approximation, an average nature of the rotation of light in a size of order $\sim \lambda$ can be obtained.

While keeping the above approximation, we insert $E_{i}=$ $u_{i} e^{i(\beta x-w t)}$ into Equation (1) and obtain the next Schrödinger equation [15] (see Appendix):

$$
\begin{aligned}
i \hbar \frac{\partial u_{i}}{\partial t} & =-\frac{\hbar^{2}}{2 n_{\mathrm{eff}}} \nabla_{2}^{2} u_{i}+V u_{i} \\
& \equiv H(t) u_{i},
\end{aligned}
$$

where we used the notations: $\hbar \equiv \lambda / 2 \pi, t \equiv x, V \equiv n_{\text {eff }}-n, \beta=$ $n_{\mathrm{eff}} k=2 \pi n_{\mathrm{eff}} / \lambda, \nabla_{2}^{2}=\partial^{2} / \partial y^{2}+\partial^{2} / \partial z^{2}$, and $n_{\mathrm{eff}}$ is the effective refractive index in the waveguide. Here we also used the slowlyvarying-envelope approximation, $\left|\partial^{2} u_{i} / \partial x^{2}\right| \ll\left|2 \beta \partial u_{i} / \partial x\right|$. In addition, we omitted a small difference in $\beta$ for waves with different polarizations (and with the same node numbers). In this situation, we can perfectly utilize ideas and descriptions in quantum mechanics to study the phenomena. Hereafter, we will deal with all quantities in units of $\hbar=c=1$, as often used in quantum mechanics.

Now we concentrate on the lowest eigenvalue of the righthand side of Equation (2) when $m=0$, as described in section 2. In this case, we denote $\bar{\Omega}$ as the lowest eigenvalue, or the lowest frequency at the crossing point (with $m=0$ ). When $m$ is added (i.e., $m \neq 0$ ), since the mode with $\bar{\Omega}$ with degeneracy splits into two modes, we set their eigenvalues to be $\Omega_{0}$ and $\Omega_{1}$, where $\Omega_{0}=\bar{\Omega}-\omega / 2$ and $\Omega_{1}=\bar{\Omega}+\omega / 2$ with a gap size of $\omega(=2 \pi f)$ between the two split modes, where $\omega$ has a large $m$-dependence but $\bar{\Omega}$ has little $m$-dependence, as already shown in Figure 5.

For those two split modes, the Hamiltonian $H(t)$ in Equation (3) can be written in the matrix representation as

$$
\begin{aligned}
H(t) & =\left(\begin{array}{cc}
\Omega_{1} & 0 \\
0 & \Omega_{0}
\end{array}\right) \\
& =\frac{\Omega_{1}+\Omega_{0}}{2} \hat{1}+\frac{\Omega_{1}-\Omega_{0}}{2} \hat{\sigma_{3}} \\
& =\bar{\Omega} \hat{1}+\omega \frac{\hat{\sigma_{3}}}{2},
\end{aligned}
$$

which is known as a two-state model [16]. In Equation (4), $\hat{1}$ is a unit matrix and $\hat{\sigma_{3}}$ is the $z$-component of the Pauli matrices $\left(\hat{\sigma_{1}}\right.$, $\left.\hat{\sigma_{2}}, \hat{\sigma_{3}}\right)$ :

$$
\hat{\sigma_{1}}=\left(\begin{array}{ll}
0 & 1 \\
1 & 0
\end{array}\right), \quad \hat{\sigma_{2}}=\left(\begin{array}{cc}
0 & -i \\
i & 0
\end{array}\right), \quad \hat{\sigma_{3}}=\left(\begin{array}{cc}
1 & 0 \\
0 & -1
\end{array}\right)
$$




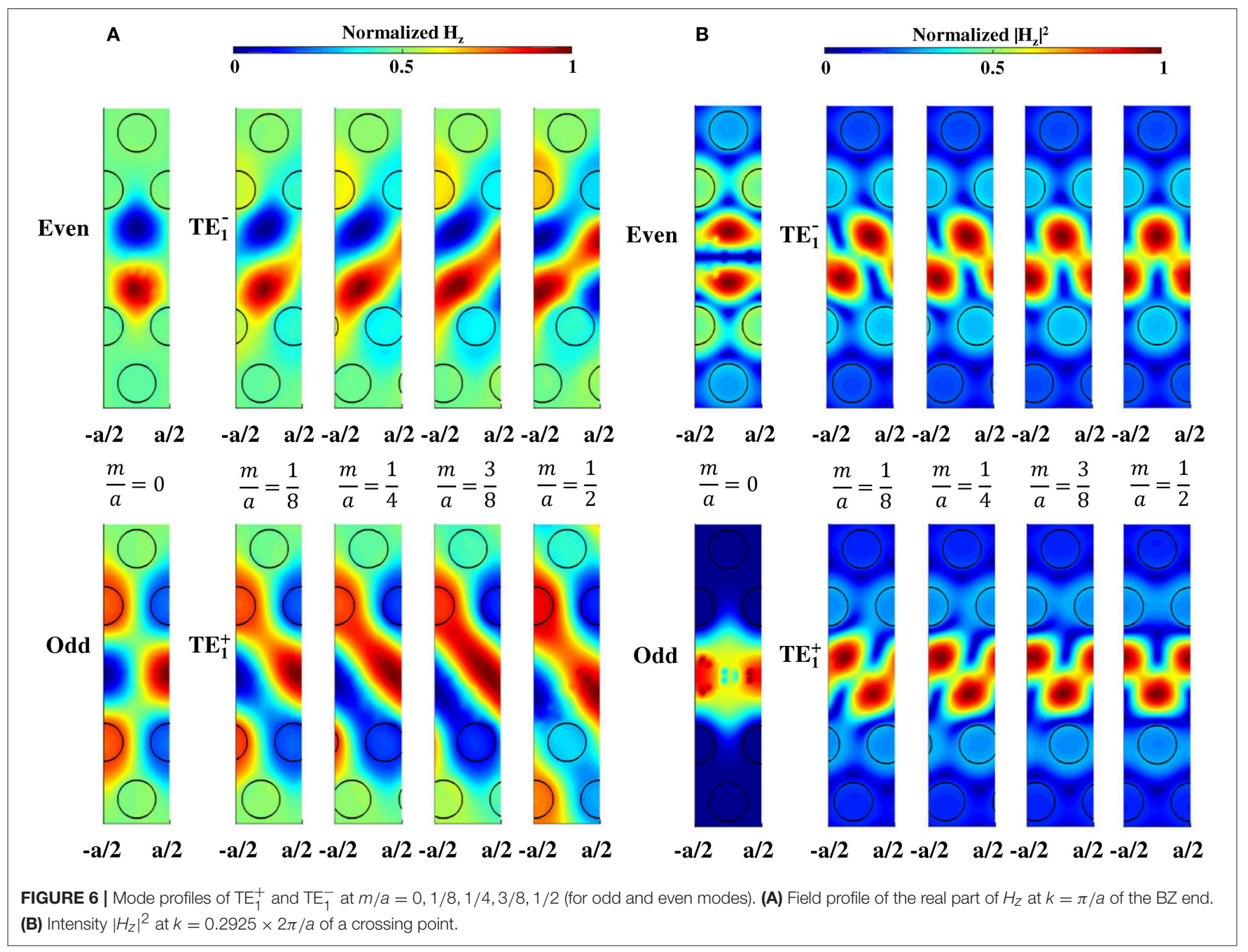

that suffice

$$
\begin{array}{r}
{\left[\hat{\sigma}_{i}, \hat{\sigma}_{j}\right]=2 i \epsilon_{i j k} \hat{\sigma}_{k},} \\
\left\{\hat{\sigma}_{i}, \hat{\sigma}_{j}\right\}=2 \delta_{i j} \hat{1}
\end{array}
$$

and thus

$$
\hat{\sigma}_{i} \hat{\sigma}_{j}=\delta_{i j} \hat{1}+i \epsilon_{i j k} \hat{\sigma_{k}}
$$

where $[X, Y]=X Y-Y X,\{X, Y\}=X Y+Y X$, and $i, j, k$ run from 1 to $3 . \epsilon_{i j k}$ is the Levi-Civita symbol, and $\delta_{i j}$ is the Kronecker delta. In what follows, we also use the vector notation of Equation (5), i.e., $\hat{\sigma}=\left(\hat{\sigma_{1}}, \hat{\sigma_{2}}, \hat{\sigma_{3}}\right)$.

For the Hamiltonian (4), the Schrödinger equation is of the form:

$$
\begin{aligned}
i \frac{d}{d t}|\phi(t)\rangle & =H(t)|\phi(t)\rangle, \\
& =\left(\bar{\Omega} \hat{1}+\omega \frac{\hat{\sigma}_{3}}{2}\right)|\phi(t)\rangle,
\end{aligned}
$$

where $|\phi(t)\rangle$ is a two-component wave function, or a spinor $[17,18]$. (Its detail is described below.) In Equation (10), using the following transformation

$$
|\phi(t)\rangle=e^{-i \bar{\Omega} \hat{1} t}|\psi(t)\rangle=e^{-i \bar{\Omega} t}|\psi(t)\rangle,
$$

we can simplify the Equation (10) as

$$
i \frac{d}{d t}|\psi(t)\rangle=\mathscr{H}(t)|\psi(t)\rangle
$$

where

$$
\mathscr{H}(t)=\omega \frac{\hat{\sigma_{3}}}{2} .
$$

In Equation (11), deriving the $e^{-i \bar{\Omega} t}$-term from $|\phi(t)\rangle$ is accepted, because we observed a sizable field/intensity tilt induced by a change in $m$ or $\omega$ (because $\omega$ has a large $m$-dependence, but not $\bar{\Omega}$ because $\bar{\Omega}$ has little $m$-dependence). Thus clearly, we can say that the tilt does not depend on $\bar{\Omega}$. Furthermore, when we calculate the expectation value for an operator $\hat{\mathscr{O}}$, we can rigorously say 
that no $\bar{\Omega}$ term contributes to the expectation value: In fact, we obtain via the transformation (11).

$$
\langle\hat{\mathscr{O}}\rangle_{t}=\langle\phi(t)|\hat{\mathscr{O}}| \phi(t)\rangle=\langle\psi(t)|\hat{\mathscr{O}}| \psi(t)\rangle .
$$

Further, at the initial state (or at $t=0$ ), the next relation holds:

$$
|\phi(0)\rangle=|\psi(0)\rangle \equiv|\psi\rangle
$$

where $|\psi\rangle$ is the (time-independent) eigenvector of Equation (13), or a spinor:

$$
|\psi\rangle=\left(\begin{array}{l}
u_{1} \\
u_{2}
\end{array}\right) .
$$

Here, $u_{1}$ and $u_{2}$ are polarized electric fields or wave functions that will come into the $\mathrm{TE}_{1}^{-}$mode and $\mathrm{TE}_{1}^{+}$mode, respectively. Equation (16) is almost the same as the Jones vector constructed with polarized electric fields, which is expressed as a spinor with an appropriate basis $[19,20]$. There is a slight difference in definition between Equation (16) and the Jones vector defined with plane EM waves, because our EM waves are the waves propagating along the $\mathrm{PhC}$ waveguide with different polarization directions; nonetheless, almost the same definition can be used (except for the polarization directions).

The above spinor of a two-state model with Equation (13) can be regarded as that of an electron with spin $\frac{1}{2}$. In this case, as is well-known, the size of $\omega$ in Equation (13) is proportional to the strength $B$ of an applied magnetic field, i.e., $\omega \propto B$ (for weak $B$ ) [16]. In this situation, we can look upon $m$ as $B$ phenomenologically by help of the "equivalence" between $\omega \propto m$ and $\omega \propto B$ (for small $m$ and $B$ ).

Next, to see the time evolution of the system with $\mathscr{H}(t)$, we use the following equation for a unitary operator $U(t)[16,21]$.

$$
i \frac{d}{d t} U(t)=\mathscr{H}(t) U(t)
$$

Equation (17) can be derived from the derivative of the identity $U(t)^{\dagger} U(t)=U(t) U(t)^{\dagger}=\hat{1}$, that is,

$$
i \frac{d U(t)}{d t} U(t)^{\dagger}=-i U(t) \frac{d U(t)^{\dagger}}{d t},
$$

which indicates that the left-hand side of Equation (18) is hermitian. If we set this hermitian part as $\mathscr{H}(t)$, Equation (17) can be obtained from Equation (18).

By inserting Equation (13) into Equation (17) and integrating Equation (17) with the initial condition $U(0)=\hat{1}$, we obtain

$$
\begin{aligned}
U(t) & =\hat{1}-i \int_{0}^{t} d t_{1} \mathscr{H}\left(t_{1}\right)+(-i)^{2} \int_{0}^{t} d t_{1} \int_{0}^{t_{1}} d t_{2} \mathscr{H}\left(t_{1}\right) \mathscr{H}\left(t_{2}\right)+\ldots \\
& =\sum_{n=0}^{\infty}(-i)^{n}\left(\omega \frac{\hat{\sigma_{3}}}{2}\right)^{n} \frac{t^{n}}{n !} \\
& =e^{-i \omega \frac{\hat{\sigma}_{3}}{2} t} \\
& =\hat{1} \cos \frac{\omega t}{2}-i \hat{\sigma}_{3} \sin \frac{\omega t}{2},
\end{aligned}
$$

where the time-integration parts have been simply calculated as

$$
\int_{0}^{t} d t_{1} \int_{0}^{t_{1}} d t_{2} \ldots \int_{0}^{t_{n-1}} d t_{n}=\frac{t^{n}}{n !}
$$

because $\mathscr{H}(t)=\omega \hat{\sigma_{3}} / 2$ has no time-dependence.

In this case, the time-evolution $|\psi(t)\rangle$ of the spinor $|\psi\rangle$ in Equation (16) is of the form:

$$
\begin{aligned}
|\psi(t)\rangle & =U(t)|\psi\rangle \\
& =e^{-i \omega \frac{\hat{\sigma}_{3}}{2} t}|\psi\rangle \\
& =\left(\hat{1} \cos \frac{\omega t}{2}-i \hat{\sigma}_{3} \sin \frac{\omega t}{2}\right)|\psi\rangle .
\end{aligned}
$$

where if we interpret that $\omega t$ is an angle $\theta$ for the polarization rotation, then Equation (26) gives a double-valuedness to $u_{2}$ (or $u_{1}$ ) in the $\theta$-rotation. Here, $t$ should be interpreted as the length of the interconnection part between the non-mismatched entrance and the mismatched exit (when we use the units of $\hbar=c=1$ ); The " $t$ " is a constant when the interconnection length has a fixed value. Even in this situation, $\theta$ can vary as $m$ changes because the insertion of $f=g f_{\max } m / a$ into $\omega=2 \pi f$ in Equation (26) gives $\theta=\omega t=2 \pi g f_{\max } t m / a=\theta_{0} m / a$, where $\theta_{0}=2 \pi g f_{\max } t$ is a constant. Inserting $\omega t=\theta$ into Equation (26), we obtain

$$
|\psi(\theta)\rangle=\left(\hat{1} \cos \frac{\theta}{2}-i \hat{\sigma_{3}} \sin \frac{\theta}{2}\right)|\psi\rangle,
$$

which provides the relation $|\psi(2 \pi)\rangle=-|\psi(0)\rangle$. Furthermore, Equation (27) can explain the aforementioned anticlockwise (clockwise) rotation of the $\mathrm{TE}_{1}^{+}\left(\mathrm{TE}_{1}^{-}\right)$mode, because the explicit expression of Equation (27) with

$$
|\psi(\theta)\rangle=\left(\begin{array}{l}
u_{1}(\theta) \\
u_{2}(\theta)
\end{array}\right), \quad|\psi\rangle=\left(\begin{array}{l}
u_{1} \\
u_{2}
\end{array}\right)
$$

is of the form:

$$
\left(\begin{array}{l}
u_{1}(\theta) \\
u_{2}(\theta)
\end{array}\right)=\left(\begin{array}{c}
e^{-i \theta / 2} u_{1} \\
e^{i \theta / 2} u_{2}
\end{array}\right)
$$

Thus, if $u_{2}(\theta)$ rotates anticlockwise, then $u_{1}(\theta)$ rotates clockwise (and vice versa). Note that in terms of $|\phi(t)\rangle$ (not $|\psi(t)|$ ), we obtain in place of Equation (29):

$$
\left(\begin{array}{l}
u_{1}(\theta) \\
u_{2}(\theta)
\end{array}\right)=\left(\begin{array}{cc}
e^{-i \Theta_{0}} & e^{-i \theta / 2} u_{1} \\
e^{-i \Theta_{0}} e^{i \theta / 2} & u_{2}
\end{array}\right)
$$

where $\Theta_{0}=\bar{\Omega} t$ is a constant when the "length" $t$ is a constant, as described above. If $m=0$, then $\theta=0$, but $\Theta_{0}$ remains as a constant (because $\bar{\Omega}$ in $\Theta_{0}$ has (almost) no $m$-dependence); in this case (or $m=0$ ), Equation (30) becomes

$$
\left(\begin{array}{l}
u_{1}(0) \\
u_{2}(0)
\end{array}\right)=\left(\begin{array}{ll}
e^{-i \Theta_{0}} & u_{1} \\
e^{-i \Theta_{0}} & u_{2}
\end{array}\right)
$$

Equation (31) corresponds to the zero- $m$ fields, as given at the leftmost ones in Figure 6A. We can see that adjusting the 
parameter " $t$ " in $\Theta_{0}$ of Equation (31) enables setting various initial polarized states at $m=0$. We should examine the field tilt for $m \neq 0$ as a difference from those initial field profiles, $\left(u_{1}(0) u_{2}(0)\right)$, and thus what we should look upon as a field tilt is given by

$$
\left(\begin{array}{l}
u_{1}(\theta) \\
u_{2}(\theta)
\end{array}\right)=\left(\begin{array}{c}
e^{-i \theta / 2} u_{1}(0) \\
e^{i \theta / 2} u_{2}(0)
\end{array}\right)
$$

and hence we obtain the same result as that in Equation (29).

As for the evolution of the angular momentum operator $\hat{\sigma}$, we can calculate it as

$$
\hat{\boldsymbol{\sigma}}(t)=U^{\dagger}(t) \hat{\boldsymbol{\sigma}} U(t) .
$$

Using Equations (21),(33), we obtain

$$
\left(\begin{array}{l}
\hat{\sigma_{1}}(t) \\
\hat{\sigma_{2}}(t) \\
\hat{\sigma_{3}}(t)
\end{array}\right)=\left(\begin{array}{ccc}
\cos \omega t & -\sin \omega t & 0 \\
\sin \omega t & \cos \omega t & 0 \\
0 & 0 & 1
\end{array}\right)\left(\begin{array}{l}
\hat{\sigma_{1}} \\
\hat{\sigma_{2}} \\
\hat{\sigma_{3}}
\end{array}\right),
$$

where Equations (8),(22) have been used in the calculations. For convenience, we rewrite Equation (34) as

$$
\hat{\sigma}_{i}(t)=a_{i j}(t) \hat{\sigma}_{j}
$$

where $j$ is a dummy index and runs from 1 to 3 . Since $\hat{\sigma}_{i}$ is defined in the $S U(2)$ space [22], it is not yet related to a vector in our real space, i.e., in the $S O(3)$ space.

Next, we show how a vector rotating in $S O(3)$ space is related to $\hat{\sigma}_{i}$ in the $S U(2)$ space. To perform this, we start with the following Schrödinger equation:

$$
\begin{array}{r}
i \frac{d}{d t}|\psi(t)\rangle=\mathscr{H}(t)|\psi(t)\rangle, \\
i \frac{d}{d t}|\psi(t)\rangle-\mathscr{H}(t)|\psi(t)\rangle=0 .
\end{array}
$$

By multiplying $\langle\psi(t)| \hat{\sigma}_{i}$ from the left to Equation (37), we obtain

$$
i\left\langle\psi(t)\left|\hat{\sigma}_{i} \frac{d}{d t}\right| \psi(t)\right\rangle-\frac{\omega}{2}\left\langle\psi(t)\left|\hat{\sigma}_{i} \hat{\sigma}_{3}\right| \psi(t)\right\rangle=0,
$$

where Equation (13) was also used. We then multiply $\hat{\sigma}_{i}|\psi(t)\rangle$ from the right to the hermitian conjugate of Equation (37) and obtain

$$
-i\left(\frac{d}{d t}\langle\psi(t)|\right) \hat{\sigma}_{i}|\psi(t)\rangle-\frac{\omega}{2}\left\langle\psi(t)\left|\hat{\sigma}_{3} \hat{\sigma}_{i}\right| \psi(t)\right\rangle=0 .
$$

By subtracting Equation (39) from Equation (38), we have

$$
\begin{aligned}
i \frac{d}{d t}\left\langle\psi(t)\left|\hat{\sigma}_{i}\right| \psi(t)\right\rangle-\frac{\omega}{2}\left\langle\psi(t)\left|\left[\hat{\sigma}_{i}, \hat{\sigma}_{3}\right]\right| \psi(t)\right\rangle & =0, \\
i \frac{d}{d t}\left\langle\psi(t)\left|\hat{\sigma}_{i}\right| \psi(t)\right\rangle+i \epsilon_{i j 3}\left\langle\psi(t)\left|\hat{\sigma}_{j}\right| \psi(t)\right| \omega & =0,
\end{aligned}
$$

where $\left[\hat{\sigma}_{i}, \hat{\sigma}_{3}\right]=2 i \epsilon_{i 3 k} \hat{\sigma}_{k}=-2 i \epsilon_{i j 3} \hat{\sigma}_{j}$ was used. In Equation (41), $\left\langle\psi(t)\left|\hat{\sigma}_{i}\right| \psi(t)\right\rangle$ is an expectation value of $\hat{\sigma}_{i}$ at "time" $t$ and is observed as a vector in the $S O(3)$ space. Also, we can show that $\left\langle\psi(t)\left|\hat{\sigma}_{i}\right| \psi(t)\right\rangle$ is actually proportional to $M_{i}(t)$ of a general vector $\boldsymbol{M}(t)$ rotating in the $S O(3)$ space, where it suffices the same-form equation as Equation (41):

$$
\frac{d}{d t} M_{i}(t)+\epsilon_{i j 3} M_{j}(t) \omega=0
$$

that is obtained [23] from

$$
\frac{d}{d t} \boldsymbol{M}(t)-\omega \times \boldsymbol{M}(t)=0
$$

where $\boldsymbol{M}(t)$ is fixed in a moving system with an angular frequency vector $\boldsymbol{\omega}=\omega \boldsymbol{e}_{3}$ and $\boldsymbol{e}_{3}$ is a unit vector in the $\boldsymbol{e}_{3}$-direction. Furthermore, in order to check the transformation property of $\left\langle\psi(t)\left|\hat{\sigma}_{i}\right| \psi(t)\right\rangle$, by use of $|\psi(t)\rangle=U(t)|\psi\rangle$, we get

$$
\begin{aligned}
\left\langle\psi(t)\left|\sigma_{i}\right| \psi(t)\right\rangle & =\left\langle\psi\left|U^{\dagger}(t) \sigma_{i} U(t)\right| \psi\right\rangle \\
& =a_{i j}(t)\left\langle\psi\left|\sigma_{j}\right| \psi\right\rangle .
\end{aligned}
$$

This means that $\left\langle\psi(t)\left|\sigma_{i}\right| \psi(t)\right\rangle$ is a vector that transforms via $a_{i j}(t)$, i.e., the rotation in $S O(3)$. In the above, we have assumed $\omega t=\theta$ since its use in Equations (27),(29). Thus, we consistently use it in the rotation of the angular momentum. We then obtain

$$
\left.\left(\begin{array}{l}
\left\langle\hat{\sigma}_{1}\right\rangle_{\theta} \\
\left\langle\hat{\sigma}_{2}\right\rangle_{\theta} \\
\left\langle\hat{\sigma}_{3}\right\rangle_{\theta}
\end{array}\right)=\left(\begin{array}{ccc}
\cos \theta & -\sin \theta & 0 \\
\sin \theta & \cos \theta & 0 \\
0 & 0 & 1
\end{array}\right)\left(\begin{array}{l}
\left\langle\hat{\sigma}_{1}\right. \\
\hat{\sigma}_{2} \\
\hat{\sigma}_{3}
\end{array}\right\rangle\right)
$$

as the explicit matrix representation of Equation (45), where $\left\langle\sigma_{i}\right\rangle=\left\langle\psi\left|\sigma_{i}\right| \psi\right\rangle$ and $\left\langle\sigma_{i}\right\rangle_{\theta}=\left\langle\psi(t)\left|\sigma_{i}\right| \psi(t)\right\rangle$ with $\omega t=\theta$ (or $\left.\left\langle\sigma_{i}\right\rangle_{\theta}=\left\langle\psi(\theta)\left|\sigma_{i}\right| \psi(\theta)\right\rangle\right)$. The relation of $\left\langle\sigma_{i}\right\rangle_{\theta}$ with the electric fields is easily obtained from the direct calculations of $\left\langle\sigma_{i}\right\rangle_{\theta}=$ $\left\langle\psi(\theta)\left|\sigma_{i}\right| \psi(\theta)\right\rangle$ with Equations (5),(28):

$$
\begin{aligned}
\left\langle\hat{\sigma}_{1}\right\rangle_{\theta} & =u_{1}^{*}(\theta) u_{2}(\theta)+u_{1}(\theta) u_{2}^{*}(\theta) \\
& =2 \operatorname{Re}\left(u_{1}^{*}(\theta) u_{2}(\theta)\right) \equiv S_{2}, \\
\left\langle\hat{\sigma}_{2}\right\rangle_{\theta} & =-i u_{1}^{*}(\theta) u_{2}(\theta)+i u_{1}(\theta) u_{2}^{*}(\theta) \\
& =2 \operatorname{Im}\left(u_{1}^{*}(\theta) u_{2}(\theta)\right) \equiv S_{3}, \\
\left\langle\hat{\sigma}_{3}\right\rangle_{\theta} & =\left|u_{1}(\theta)\right|^{2}-\left|u_{2}(\theta)\right|^{2} \equiv S_{1},
\end{aligned}
$$

where $S_{1}, S_{2}$, and $S_{3}$ are the Stokes parameters that characterize polarization rotations $[24,25]$. The electric-field intensity relates to the rest $\left(S_{0}\right)$ of the Stokes parameters:

$$
\langle\hat{1}\rangle_{\theta}=\left|u_{1}(\theta)\right|^{2}+\left|u_{2}(\theta)\right|^{2} \equiv S_{0} .
$$

Note that the rotation of the angular momentum in Equation (46) shows a single-valuedness with respect to $\theta$, which is completely different from the double-valuedness of the wave function $|\psi(\theta)|$; If we treated an electron of spin $\frac{1}{2},|\psi(\theta)\rangle$ would be the wave function of the electron, and the angular-momentum motion would correspond to spin precession [26].

In this paper, we have pointed out the "equivalence" between the $\mathrm{PhC}$ line-defect waveguide with an added phase mismatch $m$ 
and the spin-half electron with an applied magnetic field $B$. In particular, the EM wave equation in the $\mathrm{PhC}$ waveguide could be reduced to the Schrödinger equation; Both systems showed the same two-level splitting and had the split mode or level spacing in proportion to the size of the perturbation, $m$ or $B$. At the present stage of the theory with some approximations, we cannot prove the "equivalence" mathematically, but we showed it from some supporting evidence. A further development of the theory with much less approximations will be able to explain the equivalence.

\section{SUMMARY}

Using the Schrödinger equation derived from the EM wave equation, we have built a two-state model that can explain the observed polarization rotation and mode splitting that occur simultaneously when a phase mismatch $m$ is added to a PhC linedefect waveguide. The theory has given a double-valuedness to the light field (or the wave function) with different polarizations and a single-valuedness to the motion of angular momenta. Using a spinor representation, the former has explained the difference in the rotational direction (i.e., clockwise or anticlockwise direction) of $\mathrm{TE}_{1}^{-}$or $\mathrm{TE}_{1}^{+}$mode, and the latter has clarified the relation between the angular momenta and the Stokes parameters that define the polarization rotations. Also, the theory has given a relation between the rotational angle $\theta$ and the mismatch $m$

\section{REFERENCES}

1. Yablonovitch E. Inhibited spontaneous emission in solid-state physics and electronics. Phys Rev Lett. (1987) 58:2059-62.

2. John S. Strong localization of photons in certain disordered dielectric superlattices. Phys Rev Lett. (1987) 58:2486-9.

3. Yablonovitch E. Photonic crystals: semiconductors of light. Sci Am. (2001) 285:46-55. doi: 10.1038/scientificamerican1201-46

4. Joannopoulos JD, Johnson SG, Winn JN, Meade RD. Photonic Crystals Molding the Flow of Light. Princeton, NJ: Princeton University Press (2008).

5. Musslimani $\mathrm{ZH}$, Yang J. Self-trapping of light in a two-dimensional photonic lattice. J Opt Soc Am B (2004) 21:973-81. doi: 10.1364/JOSAB.21.000973

6. Bartal G, Manela O, Cohen O, Fleischer JW, Segev M. Observation of secondband vortex solitons in 2D photonic lattices. Phys Rev Lett. (2005) 95:053904. doi: 10.1103/PhysRevLett.95.053904

7. Richter T, Kaiser F. Anisotropic gap vortices in photorefractive media. Phys Rev A (2007) 76:033818 . doi: 10.1103/PhysRevA.76.033818

8. Song D, Lou C, Tang L, Wang K, Li W, Chen X, et al. Self-trapping of optical vortices in waveguide lattices with a self-defocusing nonlinearity. Opt Express (2008) 16:10110-6. doi: 10.1364/OE.16.010110

9. Wang J, Yang J. Families of vortex solitons in periodic media. Phys Rev A (2008) 77:033834. doi: 10.1103/PhysRevA.77.033834

10. Zhang Z, Ma D, Zhang Y, Cao M, Xu Z, Zhang Y. Propagation of optical vortices in a nonlinear atomic medium with a photonic band gap. Opt Lett. (2017) 42:1059-62. doi: 10.1364/OL.42.001059

11. Mills DL, Trullinger SE. Gap solitons in nonlinear periodic structures. Phys Rev B (1987) 36:947-52.

12. Chen W, Mills DL. Gap solitons and the nonlinear optical response of superlattices. Phys Rev Lett. (1987) 58:160-3.

13. Sotto M, Debnath K, Khokhar AZ, Tomita I, Thomson D, Saito S. Photonic bonding modes with circular polarization at zero-group-velocity points. In: The 15th International Conference on Group IV Photonics. Cancun:IEEE (2018).

14. Mock A, Lu L, O’Brien J. Space group theory and Fourier space analysis of two-dimensional photonic crystal waveguides. Phys Rev B (2010) 81:155115. doi: 10.1103/PhysRevB.81.155115 for small $m$ (via the numerical result between $f$ and $m$ ). In this analysis, the "equivalence" between both systems has been indicated for the light field with two split modes and the electron wave function with two split levels and for the light angular motion and the electron spin precession.

\section{AUTHOR CONTRIBUTIONS}

MS proposed the idea to introduce the mismatch. MS, IT, and SS developed theories. MS and KD conducted photonic crystal simulations. IT drafted the main text. All authors contributed to discussions.

\section{FUNDING}

This work is supported by EPSRC Standard Grant (EP/M009416/1), EPSRC Manufacturing Fellowship (EP/M008975/1), and EPSRC Platform Grant (EP/N013247/1).

\section{ACKNOWLEDGMENTS}

We are grateful to Prof. H. N. Rutt for his constructive comments. The data from the paper can be obtained from the University of Southampton ePrint research repository: doi: https://doi.org/10. 5258/SOTON/D0503.

15. Longhi S. Quantum-optical analogies using photonic structures. Laser Photon Rev. (2009) 3:243-61. doi: 10.1002/lpor.200810055

16. Sakurai JJ. Modern Quantum Mechanics. New York, NY: Addison-Wesley Publishing Co., Inc. (1985).

17. Greiner W. Quantum Mechanics: An Introduction. Heidelberg: Springer (2001).

18. Cartan E. The Theory of Spinors. Cambridge: The MIT Press (1966).

19. Castillo G, Garcia IR. The Jones vector as a spinor and its representation on the Poincare sphere. Rev Mex Fis. (2011) 57:406-13.

20. Castillo G. Spinor representation of an electromagnetic plane wave. J Phys A Math Theor. (2008) 41:115302. doi: 10.1088/1751-8113/41/11/115302

21. Takahashi Y. Mathematics for Physics I, II. Tokyo: Koudansha Co., Inc. (1993).

22. Georgi H. Lie Algebras In Particle Physics: from Isospin To Unified Theories. London: Benjamin/Cummings Publishing Co., Inc. (1982).

23. Landau LD, Lifshitz EM. Mechanics. New York, NY: Pergamon Press (1976).

24. Jackson JD. Classical Electrodynamics. New York, NY: John Wiley and Sons, Inc. (1999).

25. Milione G, Sztul HI, Nolan DA, Alfano RR. Higher-order Poincare sphere, Stokes parameters, and the angular momentum of light. Phys Rev Lett. (2011) 107:053601. doi: 10.1103/PhysRevLett.107.053601

26. Tomonaga S. The Story of Spin. Chicago, IL: University of Chicago Press (1998).

Conflict of Interest Statement: The authors declare that the research was conducted in the absence of any commercial or financial relationships that could be construed as a potential conflict of interest.

The reviewer SS and handling editor declared their shared affiliation.

Copyright (c) 2018 Sotto, Tomita, Debnath and Saito. This is an open-access article distributed under the terms of the Creative Commons Attribution License (CC BY). The use, distribution or reproduction in other forums is permitted, provided the original author(s) and the copyright owner(s) are credited and that the original publication in this journal is cited, in accordance with accepted academic practice. No use, distribution or reproduction is permitted which does not comply with these terms. 


\section{APPENDIX}

To derive the Schrödinger equation from the wave equation, we insert $E_{i}=u_{i} e^{i(\beta x-w t)}$ into

$$
\left(\nabla^{2}-\frac{n^{2}}{c^{2}} \frac{\partial^{2}}{\partial t^{2}}\right) E_{i}=0
$$

and obtain

$$
\begin{aligned}
\nabla_{2}^{2} u_{i} e^{i(\beta x-w t)}+ & \frac{\partial^{2} u_{i}}{\partial x^{2}} e^{i(\beta x-w t)}+2 i \beta \frac{\partial u_{i}}{\partial x} e^{i(\beta x-w t)} \\
& +\left(\frac{n^{2}}{c^{2}} w^{2}-\beta^{2}\right) u_{i} e^{i(\beta x-w t)}=0
\end{aligned}
$$

where $\nabla_{2}^{2}=\partial^{2} / \partial y^{2}+\partial^{2} / \partial z^{2}$ and $\beta=n_{\text {eff }} k=2 \pi n_{\text {eff }} / \lambda, k$ is the wavenumber in vacuum, and $w$ is the angular frequency.

By use of the slowly-varying-envelope approximation, $\left|\partial^{2} u_{i} / \partial x^{2}\right| \ll\left|2 \beta \partial u_{i} / \partial x\right|$, Equation (A2) is of the form:

$$
\nabla_{2}^{2} u_{i}+2 i \beta \frac{\partial u_{i}}{\partial x}+\left(\frac{n^{2}}{c^{2}} w^{2}-\beta^{2}\right) u_{i}=0
$$

By substituting $\beta=n_{\text {eff }} k$ for Equation (A3) and using an approximation, $\left|n-n_{\text {eff }}\right| \ll n+n_{\text {eff }}$, we obtain

$$
i \hbar \frac{\partial u_{i}}{\partial x}=-\frac{\hbar^{2}}{2 n_{\mathrm{eff}}} \nabla_{2}^{2} u_{i}+\left(n_{\mathrm{eff}}-n\right) u_{i}
$$

where $\lambda=\lambda / 2 \pi$. In Equation (A4), by setting $\hbar=\lambda, V=$ $n_{\text {eff }}-n$, we finally get

$$
i \hbar \frac{\partial u_{i}}{\partial t}=-\frac{\hbar^{2}}{2 n_{\mathrm{eff}}} \nabla_{2}^{2} u_{i}+V u_{i}
$$

